\title{
Observable Algebras for the Rational and Trigonometric Euler-Calogero-Moser Models
}

\author{
J. Avan * E. Billey *
}

April 1994

\begin{abstract}
We construct polynomial Poisson algebras of observables for the classical Euler-Calogero-Moser (ECM) models. The conserved Hamiltonians and symmetry algebras derived in a previous work are subsets of these algebras. We define their linear, $N \rightarrow \infty$ limits, realizing $\mathcal{W}_{\infty}$ type algebras coupled to current algebras.
\end{abstract}

PAR LPTHE 94-16

*L.P.T.H.E. Université Paris VI (CNRS UA 280), Box 126, Tour 16, $1^{\text {er }}$ étage, 4 place Jussieu, F-75252 PARIS CEDEX 05 


\section{Introduction}

The Calogero-Moser (CM) models [1] and their extensions to internal degrees of freedom known as EulerCalogero-Moser (ECM) models [2] have received a lot of attention in the past year. In particular, remarkable algebraic structures were identified when considering specific sets of invariant functions of the Lax matrices associated with these models. In the case of CM models, polynomial Poisson-bracket algebras of observables were constructed [3] for rational and trigonometric potentials; their $N \rightarrow \infty$ limit realized respectively the classical linear $\mathcal{W}$ algebras $\operatorname{Sdiff}\left(\mathbb{R}^{2}\right)$ and $\operatorname{Sdiff}\left(\mathbb{R} \times \mathrm{S}^{1}\right)$, 3 , $\mathbb{A}$. ECM models were shown to have symmetry algebras: exact current algebras $\widehat{s l}(r)$ [2] - in the rational case - and exact Yangian algebras (quadratic deformations of $\widehat{s l}(r))$ - in the trigonometric case 5 . These algebras were closely connected to the quantum algebra obtained for the Haldane-type spin chain [6].

More recently, it was suggested that a set of observables for the quantum ECM models, realizing a $\mathcal{W}_{\infty}$ algebra when $N \rightarrow \infty$, could be used to obtain the spectrum of the theory [7]. The finite $N$ case is however not so clear. This lead us to consider in detail possible extensions of the previously known classical exact current and Yangian algebras for finite $N$, using the techniques previously developed to build the standard $\mathrm{CM}$ observable algebra [3]. We shall here describe general algebras of classical observables, associated both to the rational and trigonometric ECM models; we shall compute the Poisson-bracket structures, and describe their $N \rightarrow \infty$ limit in connection with the considerations in [7].

At this point, and before delving into the explicit construction of the observable algebras, we need to state a keystone result:

Proposition 1 Given a phase space $\mathcal{M}$ and a set of matrices $L_{(i)}$ on $\mathcal{M}$, taking values in a Lia algebra $\mathcal{G}$ and realizing the Poisson bracket structure:

$$
\left\{L_{(i)} \stackrel{\otimes}{,} L_{(j)}\right\}=\left[R, L_{(i)} \otimes \mathbb{1}\right]-\left[R^{\pi}, \mathbb{1} \otimes L_{(j)}\right]
$$

with $R \in \mathcal{G} \otimes \mathcal{G}$, the Poisson brackets of invariants

$$
\left\{\operatorname{Tr}\left(L_{(1)}^{n_{1}} \cdots L_{(p)}^{n_{p}}\right), \operatorname{Tr}\left(L_{(1)}^{m_{1}} \cdots L_{(q)}^{m_{q}}\right)\right\}
$$

vanish exactly.

Proof. The expression (2) takes the form:

$$
\begin{aligned}
& \left\{\operatorname{Tr}\left(L_{(1)}^{n_{1}} \cdots L_{(p)}^{n_{p}}\right), \operatorname{Tr}\left(L_{(1)}^{m_{1}} \cdots L_{(q)}^{m_{q}}\right)\right\} \\
= & \sum_{i=1}^{p} \sum_{j=1}^{q} \sum_{a=1}^{n_{i}} \sum_{b=1}^{m_{j}} \operatorname{Tr}\left[L_{(1)}^{n_{1}} \cdots L_{(i-1)}^{n_{i-1}} L_{(i)}^{n_{i}-a} \otimes L_{(1)}^{m_{1}} \cdots L_{(j-1)}^{m_{j-1}} L_{(j)}^{m_{j}-b}\left(\left[R, L_{(i)} \otimes 1\right]-\left[R^{\pi}, 1 \otimes L_{(j)}\right]\right)\right. \\
= & \left.\sum_{(i)}^{a-1} L_{(i+1)}^{n_{i+1}} \cdots L_{(p)}^{n_{p}} \otimes L_{(j)}^{b-1} L_{(j+1)}^{m_{j+1}} \cdots L_{(q)}^{m_{q}}\right] \\
& \sum_{j=1}^{p} \sum_{a=1}^{q} \sum_{b=1}^{n_{i}} \operatorname{Tr}\left[L_{(i)}^{a-1} L_{(i+1)}^{m_{j}} \cdots L_{(p)}^{n_{p}} L_{(1)}^{n_{1}} \cdots L_{(i-1)}^{n_{i-1}} L_{(i)}^{n_{i}-a} \otimes L_{(j)}^{b-1} L_{(j+1)}^{m_{j+1}} \cdots L_{(q)}^{m_{q}} L_{(1)}^{m_{1}} \cdots L_{(j-1)}^{m_{j-1}} L_{(j)}^{m_{j}-b}\right. \\
& \left.\left(R L_{(i)} \otimes \mathbb{1}-L_{(i)} \otimes \mathbb{1} R\right)\right] \quad+\left[R^{\pi}-\text { contribution }\right]
\end{aligned}
$$

by cyclicity of the trace. Eliminating the terms $-L_{(i)}^{a-1}(\cdots) L_{(i)}^{n_{i}-a}+L_{(i)}^{a}(\cdots) L_{(i)}^{n_{i}-a-1}$ when $a$ goes from 1 to $n_{i}$ leaves us with only the extreme terms:

$$
\begin{aligned}
& \sum_{i=1}^{p} \sum_{j=1}^{q} \sum_{b=1}^{m_{j}} \operatorname{Tr}\left(L_{(i)}^{n_{i}} \cdots L_{(p)}^{n_{p}} L_{(1)}^{n_{1}} \cdots L_{(i-1)}^{n_{i-1}} \otimes L_{(j)}^{b-1} L_{(j+1)}^{m_{j+1}} \cdots L_{(q)}^{m_{q}} L_{(1)}^{m_{1}} \cdots L_{(j-1)}^{m_{j-1}} L_{(j)}^{m_{j}-b} R\right) \\
- & \sum_{i=1}^{p} \sum_{j=1}^{q} \sum_{b=1}^{m_{j}} \operatorname{Tr}\left(L_{(i+1)}^{n_{i+1}} \cdots L_{(p)}^{n_{p}} L_{(1)}^{n_{1}} \cdots L_{(i)}^{n_{i}} \otimes L_{(j)}^{b-1} L_{(j+1)}^{m_{j+1}} \cdots L_{(q)}^{m_{q}} L_{(1)}^{m_{1}} \cdots L_{(j-1)}^{m_{j-1}} L_{(j)}^{m_{j}-b} R\right) \\
+ & {\left[R^{\pi}-\text { contribution }\right] . }
\end{aligned}
$$


For fixed values of $j$ and $b$, the first-space terms cancel two by two by obvious cyclicity of their form. Similarly the $R^{\pi}$ terms vanish by the exact cyclic structure of the second-space terms in their contribution. $\square$

This result generalizes in a straightforward way the standard theorem for a single Lax matrix with an $r$-matrix Poisson-structure [8]. It can be reexpressed in a form which will be more useful for us:

Proposition 2 Given a Lax matrix $L$ and a set of dynamical matrices $A_{(i)}$ realizing the Poisson algebra:

$$
\begin{aligned}
\{L \stackrel{\otimes}{,} L\} & =[R, L \otimes \mathbb{1}]-\left[R^{\pi}, \mathbb{1} \otimes L\right]+\text { extra terms } \\
\left\{L \stackrel{\otimes}{,} A_{(i)}\right\} & =-\left[R^{\pi}, \mathbb{1} \otimes A_{(i)}\right]+\text { extra terms } \\
\left\{A_{(i)} \stackrel{\otimes}{,} A_{(j)}\right\} & =\text { terms }
\end{aligned}
$$

the algebraic structure of the polynomial set $\operatorname{Tr}\left(L^{n} A_{(1)}^{m_{1}} \cdots A_{(p)}^{m_{p}}\right)$ is given solely by the extra terms in (3).

Proof. Apply prop.(1]) to the set $\left\{L_{(i, \pm)}\right\}=\left\{L \pm A_{(i)}\right\}$ which realize the Poisson structure (11) if $\left\{L, A_{(i)}\right\}$ realize (3) without extra terms and substitute $A_{(i)}=\frac{1}{2}\left(L_{(i,+)}-L_{(i,-)}\right)$. $\square$

\section{The rational Euler-Calogero-Moser model}

The system consists of $N$ particles on a line with pairwise $1 / r^{2}$ interactions depending of their internal degrees of freedom. The phase space is described by $2 N$ conjugate coordinates $\left(p_{i}, q_{i}\right)_{i=1 \cdots N}$ and $2 N r$ internal conjugate coordinates $\left(\eta_{i}^{a}, \xi_{i}^{a}\right)_{i=1 \cdots N, a=1 \cdots r}$ parametrizing a coadjoint orbit in $s l(N)$ as $F_{i j}=\sum_{a=1}^{r} \xi_{i}^{a} \eta_{j}^{a}$. Integrability of the model requires to restrict oneself to the submanifold $F_{i i}=\sum_{a=1}^{r} \xi_{i}^{a} \eta_{i}^{a}=\alpha$. The original Hamiltonian is:

$$
H=\frac{1}{2} \sum_{i=1}^{N} p_{i}^{2}-\frac{1}{2} \sum_{\substack{i, j=1 \\ i \neq j}}^{N} \frac{F_{i j} F_{j i}}{\left(q_{i}-q_{j}\right)^{2}} .
$$

The equations of motion with spectral parameter $\lambda$ take the Lax form $\dot{L}(\lambda)=[L(\lambda), M]$ with the Lax pair:

$$
\begin{aligned}
L(\lambda) & =L-\frac{1}{\lambda} F=\sum_{i=1}^{N} p_{i} e_{i i}-\sum_{\substack{i, j=1 \\
i \neq j}}^{N} \frac{F_{i j}}{q_{i}-q_{j}} e_{i j}-\frac{1}{\lambda} \sum_{i, j=1}^{N} F_{i j} e_{i j} \\
M & =\sum_{\substack{i, j=1 \\
i \neq j}}^{N} \frac{F_{i j}}{\left(q_{i}-q_{j}\right)^{2}} e_{i j}
\end{aligned}
$$

Our algebra of observables will consist of traces of monomials of the set

$$
\left\{L, F^{a b}=\sum_{i, j=1}^{N} \xi_{i}^{b} \eta_{j}^{a} e_{i j}, Q=\sum_{i=1}^{N} q_{i} e_{i i}\right\} .
$$

This choice is the generalization to the ECM model of the observable algebras considered in the CM models [3]. The choice of invariant quantities as observables is natural since the ECM model, reduced to orbits $\left(\xi_{i}^{a}, \eta_{i}^{a}\right)$, is a Hamiltonian reduction of a matrix-valued model [2, 9], and the adjoint-invariant quantities are precisely those which survive without modification the conjugation which redefines the relevant reduced variables. These matrices realize the following Poisson structure [10:

$$
\begin{aligned}
\{L \stackrel{\otimes}{,} L\} & =[R, L \otimes \mathbb{1}]-\left[R^{\pi}, \mathbb{1} \otimes L\right]-\sum_{\substack{i, j=1 \\
i \neq j}}^{N} \frac{F_{i i}-F_{j j}}{\left(q_{i}-q_{j}\right)^{2}} e_{i j} \otimes e_{j i} \\
\{L \stackrel{\otimes}{,} Q\} & =-\left[R^{\pi}, \mathbb{1} \otimes Q\right]+\mathcal{C} \\
\left\{L \stackrel{\otimes}{,} F^{a b}\right\} & =-\left[R^{\pi}, \mathbb{1} \otimes F^{a b}\right] \\
\{Q \stackrel{\otimes}{,} Q\} & =\left\{Q \stackrel{\otimes}{,} F^{a b}\right\}=0 \\
\left\{F^{a b} \stackrel{\otimes}{,} F^{c d}\right\} & =\left(\delta_{a d} F^{c b} \otimes \mathbb{1}-\delta_{c b} \mathbb{1} \otimes F^{a d}\right) \mathcal{C}
\end{aligned}
$$


where

$$
R=-\sum_{\substack{i, j=1 \\ i \neq j}}^{N} \frac{1}{q_{i}-q_{j}} e_{i j} \otimes e_{j i}
$$

and

$$
\mathcal{C}=\sum_{i, j=1}^{N} e_{i j} \otimes e_{j i}
$$

is the quadratic Casimir of the $\operatorname{sl}(N)$ algebra.

We are therefore in the situation described by prop.(2), guaranteeing cancellation of the $R$-matrix contributions when computing the Poisson structure of the monomial traces of $L, Q, F^{a b}$. Moreover, as usual, we may also ignore the contribution $\left(F_{i i}-F_{j j}\right)$ once we restrict the system to the manifold $F_{i i}=\alpha$. Indeed, $F_{i i}$ generates the conjugation of all matrices $L, F^{a b}$ and $Q$ (trivially) by $U(1)$ acting on the $i^{\text {th }}$ vector of the basis. Hence the Poisson brackets of $F_{i i}$ with (adjoint-invariant) traces always vanish, and it is consistent to compute the algebra of such quantities on the sub-manifold $F_{i i}=\alpha$.

Furthermore, the generators $L$ and $Q$ obey the commutation relation:

$$
[L, Q]=F-\sum_{i=1}^{N} F_{i i} e_{i i}=F-\alpha \mathbb{1} .
$$

We now describe the algebra of observables, using the following properties.

Proposition 3 Given two matrices $A, B$, the monomial $\operatorname{Tr}\left(A F^{a b} B F^{c d}\right)$ is rewritten as $\operatorname{Tr}\left(A F^{c b}\right) \operatorname{Tr}\left(B F^{a d}\right)$.

It follows that we shall only retain monomials of order 0 or 1 in $F^{a b}$. The proof of prop.(3) is obvious, relying on the projector structure of $F^{a b}$ as $F_{i j}^{a b}=\xi_{i}^{b} \eta_{j}^{a}$.

Proposition 4 All monomials of the form $\operatorname{Tr}\left(A_{(1)} \cdots A_{(n)}\right)$ and $\operatorname{Tr}\left(A_{(1)} \cdots A_{(n)} F^{a b}\right)$ with $A_{(i)} \in\{L, Q\}$ can be written as polynomials of normal-ordered generators $\operatorname{Tr}\left(L^{p} Q^{q}\right)$ and $\operatorname{Tr}\left(L^{p} Q^{q} F^{a b}\right), p, q \in \mathbb{N}$.

Proof. As in [3] the commutation relation (8) and the projective property (3) allow a recursive proof of prop.(价. Specifically, denoting by $l$ the length of a monomial, i.e., the total number of $L$ and $Q$ generators, one has:

- For $l=1$, normal-ordering is immediate.

- If normal-ordering is ensured up to the length $l_{0}-1$, consider first $\operatorname{Tr}(L \cdots Q)$ of length $l_{0}$. If it is already normal-ordered, the procedure stops; if not, normal-ordering is achieved by commuting $Q$ 's through $L$ 's. Each such step eliminates two generators $L, Q$ and creates one generator $F-\alpha \mathbb{1}$, thereby leaving residual terms of length $l_{0}-2$ to which the recursion hypothesis applies. Note that, had we allowed $Q^{-1}$ as a generator, the relevant commutation relation $\left[L, Q^{-1}\right]=-Q^{-1}(F-\alpha \mathbb{1}) Q^{-1}$ prevents the normal-ordering recursion. This will not occur in the trigonometric case.

Consider now $\operatorname{Tr}\left(L \cdots Q F^{a b}\right)$. Each commutation operation eliminates again two generators $L, Q$ and creates one generator $F-\alpha \mathbb{1}$. The projection property (3) allows then factorization of the residual terms into terms with one single $F^{c d}$ generator, of length at most $l_{0}-2$, to which the recursion property then applies.

Note that, as in [3], the normal-ordering of a given monomial may not be unique; due to the particular form of the matrices and their finite size, degeneracies will occur; in any explicit computation, they will be fixed at every order by the choice of a reordering path for a given monomial.

Proposition 5 The quantities $\operatorname{Tr}\left(L^{p} Q^{q} F\right)$ can be rewritten as polynomials of the variables $\operatorname{Tr}\left(L^{n} Q^{m}\right)$ and $\operatorname{Tr}\left(L^{n} Q^{m} \tilde{F}^{a b}\right)$ where $\tilde{F}^{a b}=F^{a b}-\delta_{a b} / r F$.

Proof. Again by recursion on the length $l=p+q$. 
- When the length is 0 or 1 ,

$$
\begin{aligned}
\operatorname{Tr}(F) & =N \alpha \\
\operatorname{Tr}(L F) & =\operatorname{Tr}(L([L, Q]+\alpha \mathbb{1}))=\alpha \operatorname{Tr}(L) \\
\operatorname{Tr}(Q F) & =\operatorname{Tr}(Q([L, Q]+\alpha \mathbb{1}))=\alpha \operatorname{Tr}(Q) .
\end{aligned}
$$

- Assuming that the proposition stands up to the length $l_{0}-1$, we take $n+m=l_{0}$. Then

$$
0=\operatorname{Tr}\left[L, L^{n} Q^{m}\right]=m \operatorname{Tr}\left(L^{n} Q^{m-1}(F-\alpha \mathbb{1})\right)+\text { reordering terms of } L^{n} Q^{m-1} F .
$$

Reordering terms of $L^{n} Q^{m-1} F$ contain two $F$ 's and at most $l_{0}-2$ terms $L$ and $Q$. Hence they factorize into terms linear in $F^{a b}$, following prop.(3), and the factors normal-order following prop.(俰). Finally every factor $\operatorname{Tr}\left(L^{p} Q^{q} F^{a b}\right)$ is rewritten as $\operatorname{Tr}\left(L^{p} Q^{q} \tilde{F}^{a b}\right)+\delta_{a b} / r \operatorname{Tr}\left(L^{p} Q^{q} F\right)$ with $p+q \leq l_{0}-2$, to which the recursion hypothesis applies. $\square$

It follows that the generators of our observable algebra to be considered are reduced to the set:

$$
\begin{aligned}
W_{n}^{m} & =\operatorname{Tr}\left(L^{n} Q^{m}\right) \\
\tilde{F}_{n, m}^{a b} & =\operatorname{Tr}\left(L^{n} Q^{m} \tilde{F}^{a b}\right) .
\end{aligned}
$$

The Poisson algebra (7) is slightly modified to take into account the change of generators:

$$
\begin{aligned}
\left\{\tilde{F}^{a b} \stackrel{\otimes}{,} \tilde{F}^{c d}\right\}= & \quad\left(\delta_{a d} \tilde{F}^{c b} \otimes \mathbb{1}-\delta_{c b} \mathbb{1} \otimes \tilde{F}^{a d}\right) \mathcal{C} \\
- & \frac{\delta_{a b}}{r}\left(\tilde{F}^{c d} \otimes \mathbb{1}-\mathbb{1} \otimes \tilde{F}^{c d}\right) \mathcal{C}-\frac{\delta_{c d}}{r}\left(\tilde{F}^{a b} \otimes \mathbb{1}-\mathbb{1} \otimes \tilde{F}^{a b}\right) \mathcal{C} \\
+ & \frac{1}{r}\left(\delta_{a d} \delta_{c b}-\frac{1}{r} \delta_{a b} \delta_{c d}\right)(F \otimes \mathbb{1}-\mathbb{1} \otimes F) \mathcal{C} .
\end{aligned}
$$

The Poisson algebra of observables then follows from prop.(2), eq.(7) and (11) and prop.(3) and (4).

$$
\begin{aligned}
\left\{W_{n_{1}}^{m_{1}}, W_{n_{2}}^{m_{2}}\right\}= & \sum_{i=1}^{n_{1}} \sum_{j=1}^{m_{2}} \operatorname{Tr}\left(L^{n_{1}-i} Q^{m_{1}} L^{i-1} Q^{m_{2}-j} L^{n_{2}} Q^{j-1}\right) \\
& -\sum_{i=1}^{m_{1}} \sum_{j=1}^{n_{2}} \operatorname{Tr}\left(Q^{m_{1}-i} L^{n_{1}} Q^{i-1} L^{n_{2}-j} Q^{m_{2}} L^{j-1}\right) \\
= & \left(n_{1} m_{2}-n_{2} m_{1}\right) W_{n_{1}+n_{2}-1}^{m_{1}+m_{2}-1}+\text { ordering terms } \\
\left\{W_{n_{1}}^{m_{1}}, \tilde{F}_{n_{2}, m_{2}}^{a b}\right\}= & \sum_{i=1}^{n_{1}} \sum_{j=1}^{m_{2}} \operatorname{Tr}\left(L^{n_{2}} Q^{j-1} L^{n_{1}-i} Q^{m_{1}} L^{i-1} Q^{m_{2}-j} \tilde{F}^{a b}\right) \\
& -\sum_{i=1}^{m_{1}} \sum_{j=1}^{n_{2}} \operatorname{Tr}\left(L^{j-1} Q^{m_{1}-i} L^{n_{1}} Q^{i-1} L^{n_{2}-j} Q^{m_{2}} \tilde{F}^{a b}\right) \\
= & \left(n_{1} m_{2}-n_{2} m_{1}\right) \tilde{F}_{n_{1}+n_{2}-1, m_{1}+m_{2}-1}^{a b} \text { ordering terms }
\end{aligned}
$$

the ordering terms being of length $\leq n_{1}+n_{2}+m_{1}+m_{2}-4$.

$$
\begin{aligned}
\left\{\tilde{F}_{n_{1}, m_{1}}^{a b}, \tilde{F}_{n_{2}, m_{2}}^{c d}\right\}= & \sum_{i=1}^{n_{1}} \sum_{j=1}^{m_{2}} \operatorname{Tr}\left(L^{n_{1}-i} Q^{m_{1}} \tilde{F}^{a b} L^{i-1} Q^{m_{2}-j} \tilde{F}^{c d} L^{n_{2}} Q^{j-1}\right) \\
& -\sum_{i=1}^{m_{1}} \sum_{j=1}^{n_{2}} \operatorname{Tr}\left(Q^{m_{1}-i} \tilde{F}^{a b} L^{n_{1}} Q^{i-1} L^{n_{2}-j} Q^{m_{2}} \tilde{F}^{c d} L^{j-1}\right) \\
& +\delta_{a d} \operatorname{Tr}\left(L^{n_{2}} Q^{m_{2}} L^{n_{1}} Q^{m_{1}} \tilde{F}^{c b}\right)-\delta_{c b} \operatorname{Tr}\left(L^{n_{2}} Q^{m_{2}} L^{n_{1}} Q^{m_{1}} \tilde{F}^{a d}\right) \\
& +\frac{1}{r} \operatorname{Tr}\left\{\left[L^{n_{1}} Q^{m_{1}}, L^{n_{2}} Q^{m_{2}}\right]\left(\delta_{a b} \tilde{F}^{c d}+\delta_{c d} \tilde{F}^{a b}-\left(\delta_{a d} \delta_{c b}-\frac{1}{r} \delta_{a b} \delta_{c d}\right) F\right)\right\} .
\end{aligned}
$$


The last term in (14) generates a sub-leading contribution with respect to the third term. The factorization property (3) is now replaced by:

$$
\begin{aligned}
\operatorname{Tr}\left(A \tilde{F}^{a b} B \tilde{F}^{c d}\right)= & \operatorname{Tr}\left(A F^{c b}\right) \operatorname{Tr}\left(B F^{a d}\right)-\frac{\delta_{a b}}{r} \sum_{i=1}^{r} \operatorname{Tr}\left(A F^{c i}\right) \operatorname{Tr}\left(B F^{i d}\right)-\frac{\delta_{c d}}{r} \sum_{i=1}^{r} \operatorname{Tr}\left(A F^{i b}\right) \operatorname{Tr}\left(B F^{a i}\right) \\
+ & \frac{\delta_{a b} \delta_{c d}}{r^{2}} \sum_{i, j=1}^{r} \operatorname{Tr}\left(A F^{i j}\right) \operatorname{Tr}\left(B F^{j i}\right)
\end{aligned}
$$

which is then converted into a canonical expression in terms of $\operatorname{Tr}\left(A \tilde{F}^{a b}\right)$ by using prop.(5). From prop.(5) we know that normal-order reexpresses $\operatorname{Tr}\left(L^{n} Q^{m} F\right)$ as $\alpha \operatorname{Tr}\left(L^{n} Q^{m}\right)+$ lower order polynomials. Hence, keeping only the explicit highest order of each term in (14) we get:

$$
\begin{aligned}
\left\{\tilde{F}_{n_{1}, m_{1}}^{a b}, \tilde{F}_{n_{2}, m_{2}}^{c d}\right\}= & \sum_{i=1}^{n_{1}} \sum_{j=1}^{m_{2}} \tilde{F}_{n_{1}+n_{2}-i, m_{1}+j}^{c b} \tilde{F}_{i-1, m_{2}-j-1}^{a d}+S_{1} \\
& -\sum_{i=1}^{m_{1}} \sum_{j=1}^{n_{2}} \tilde{F}_{i, m_{1}-j}^{c b} \tilde{F}_{n_{1}+n_{2}-i, m_{2}+j}^{a d}+S_{2} \\
& +\delta_{a d} \tilde{F}_{n_{1}+n_{2}, m_{1}+m_{2}}^{c b}-\delta_{c b} \tilde{F}_{n_{1}+n_{2}, m_{1}+m_{2}}^{a d}+S_{3} \\
& +S_{4} .
\end{aligned}
$$

$S_{1}$ and $S_{2}$ contain the extra terms in (15) due to the redefinition of $\tilde{F}$, plus ordering terms. $S_{3}$ contains reordering terms, and $S_{4}$ contains the purely reordering terms arising from the commutator- like term in (14), consequence of the Poisson-bracket modification (11).

At this point a number of remarks are to be made:

1. No reordering terms. A number of Poisson-brackets (12, 13, 16) have no extra reordering terms. This is true each time the second term in the Poisson-bracket expression contains only one generator $L, Q$ at any power and another generator $L, Q, \tilde{F}^{a b}$ at power 1 . One has:

$$
\begin{aligned}
& \left\{W_{n}^{0}, W_{m}^{0}\right\}=0 \quad(N \text { first commuting ECM Hamiltonians [2, 10] }) \\
& \left\{W_{n}^{0}, W_{m}^{1}\right\}=m W_{n+m-1}^{0} \quad\left(W_{m}^{1}\right. \text { as "intertwiner" between Hamiltonians) } \\
& \left\{W_{n}^{1}, W_{m}^{1}\right\}=(n-m) W_{n+m-1}^{1} \quad \text { (centerless Virasoro algebra, mentioned in [7]). }
\end{aligned}
$$

This closed set generates a symmetric Lie algebra. Note that, as a curiosity, one also has:

$$
\left\{W_{n}^{2}, W_{m}^{0}\right\}=2 m W_{n+m-1}^{1} .
$$

The current algebra symmetry also belongs to this class [2, 5]:

$$
\begin{aligned}
& \left\{W_{n}^{0}, F_{m, 0}^{a b}\right\}=0 \\
& \left\{F_{m 0}^{a b}, F_{n, 0}^{c d}\right\}=\delta_{a d} F_{n+m, 0}^{c b}-\delta_{c b} F_{n+m, 0}^{a d} .
\end{aligned}
$$

The Virasoro algebra acts on the current algebra as:

$$
\left\{W_{n}^{1}, F_{m, 0}^{a b}\right\}=-m F_{n+m-1,0}^{a b} .
$$

No extra reordering terms also occurs in a different class of Poisson brackets:

$$
\left\{W_{n}^{0}, W_{0}^{m}\right\}=n m W_{n-1}^{m-1}
$$

This structure is related, as we shall see, to the classical version of the construction of the observables in $|7|$. 
2. Different choices of basis. Absence of normal-ordering contributions also occur when computing Poisson-brackets of very specific polynomials of the original generators $W, \tilde{F}$; they correspond in fact to relevant non-linear changes of basis: the first typical example consists of the higher Hamiltonians. Only the $N$ first Hamiltonians are simple generators $W_{n}^{0}$; the higher ones are polynomials in $L$ and $\tilde{F}$, typically Weyl-ordered monomials of $L^{n}$ and $F^{m}$

$$
H_{n}^{m}=\oint \frac{d \lambda}{2 \mathrm{i} \pi \lambda^{m+1}}(L+\lambda F)^{n+m} .
$$

Since $F=\sum_{a=1}^{r} F^{a a}$, the conserved higher Hamiltonians are particular polynomials in $\operatorname{Tr}\left(L^{n} \tilde{F}^{a b}\right)$, necessarily scalar under $s l(r)$, and their commutation follows much more easily from direct computation using the spectral-parameter Lax operator 10].

Another set of exactly Poisson-commuting Hamiltonians can be obtained by direct construction, following the procedure in [3]. We define:

$$
L^{ \pm}=L \pm Q \pm \omega Q^{2} \quad(\omega \in \mathbb{R}) .
$$

From the Poisson structure (7), and repeating the derivation in [3] one gets:

$$
\begin{aligned}
\left\{L^{+} L^{-} \stackrel{\otimes}{,} L^{+} L^{-}\right\}= & {\left[\mathcal{R}, L^{+} L^{-} \otimes \mathbb{1}\right]-\left[\mathcal{R}^{\pi}, \mathbb{1} \otimes L^{+} L^{-}\right] } \\
- & 2 \mathcal{C}\left(L^{+} L^{-} \otimes \mathbb{1}-\mathbb{1} \otimes L^{+} L^{-}\right) \\
- & 2 \omega \mathcal{C}\left(L^{+} Q L^{-} \otimes \mathbb{1}-\mathbb{1} \otimes L^{+} Q L^{-}+L^{+} L^{-} \otimes Q-Q \otimes L^{+} L^{-}\right)
\end{aligned}
$$

with

$$
\mathcal{R}=\left(\mathbb{1} \otimes L^{+}\right) R+R\left(\mathbb{1} \otimes L^{-}\right) .
$$

Although not an exact $r$-matrix structure, this nevertheless guarantees the commutation of the $N$ first Hamiltonians $H^{(n)}=1 / 2 n \operatorname{Tr}\left(L^{+} L^{-}\right)^{n}$. In particular this set includes a natural one-body extension of the rational ECM Hamiltonian:

$$
H^{(1)}=\frac{1}{2} \sum_{i=1}^{N} p_{i}^{2}-\frac{1}{2} \sum_{\substack{i, j=1 \\ i \neq j}}^{N} \frac{F_{i j} F_{j i}}{\left(q_{i}-q_{j}\right)^{2}}-\frac{1}{2} \sum_{i=1}^{N}\left(q_{i}^{2}+2 \omega q_{i}^{3}+\omega^{2} q_{i}^{4}\right) .
$$

This extension was indeed considered in [11] and a set of $N$ commuting Hamiltonians was constructed. Note that full integrability would require constructing an extra set of $N(r-1)$ commuting Hamiltonians, which we do not see clearly how to get at the moment.

The quantities $\operatorname{Tr}\left(L^{+} L^{-}\right)^{n}$ naturally belong to our algebra of observables. They are however not naturally normal-ordered in terms of $L$ and $Q$, hence they are expressed as complicated polynomials of our generators - except $H^{(1)}=1 / 2 \operatorname{Tr}\left(L^{2}+Q^{2}+2 \omega Q^{3}+\omega^{2} Q^{4}\right)$. Obviously the associated algebra of observables from which to deduce exact eigenfunctions of the Hamiltonian flows should be extracted from the $\left\{L^{+}, L^{-}\right\}$representation. This represents a non-linear change of variables; but the identical normal-ordering procedure (in terms of $L^{ \pm}$) and algebra structure will be obtained in a similar way to the case of $L, Q$ since the Poisson structure and commutation relations are essentially of the same form. Suitability of the basis depends on which problem - precisely which Hamiltonians - one considers. A similar connection relates the algebras obtained in [3] and [- $[$ for the CM model.

3. The Hikami-Wadati algebra. The algebra considered in [7] consisted of generators obtained by successively commuting the quantum Hamiltonians by the operator $\operatorname{Tr}\left(Q^{2}\right)$. These quantum Hamiltonians are in fact obtained from expanding the quantum determinant of the generating transfer matrix [6]. It is to be expected that their classical limit is equivalently described by the Poisson-commuting classical traces $\operatorname{Tr}\left(L^{n}\right)$. Hence the classical construction of the Hikami-Wadati algebra is achieved by bracketing $\operatorname{Tr}\left(L^{n}\right)$ by $\operatorname{Tr}\left(Q^{2}\right)$, the first step of which is one of the Poisson-brackets (21). This is easily seen to substitute at each step one term $p_{i}$ into one term $q_{i}$, hence leading to a set of the form:

$$
W_{H W}^{n,(s)}=\frac{1}{C_{n+2(s-1)}^{s-1}} \oint \frac{d \omega}{2 \mathrm{i} \pi \omega^{s}} \operatorname{Tr}(L+\omega Q)^{n+2(s-1)} .
$$


This follows from the fact that for any matrices $A, B$,

$$
\left\{\operatorname{Tr}(A L B), \operatorname{Tr}\left(Q^{2}\right)\right\}=\operatorname{Tr}(A Q B)+\text { contributions from } A, B .
$$

Hence the $W_{H W}^{n,(s)}$ are a set of "Weyl-ordered" generators. However, for $N$ finite, they do not close an algebra. Indeed

$$
\left\{W_{H W}^{1,(2)}, W_{H W}^{1,(3)}\right\}=W_{H W}^{2,(3)}+\frac{3}{10}\left[-5 \alpha^{2} W_{H W}^{2,(1)}+3 \sum_{a, b=1}^{r} \operatorname{Tr}\left(L^{2} F^{a b}\right) \operatorname{Tr}\left(F^{b a}\right)+2 \sum_{a, b=1}^{r} \operatorname{Tr}\left(L F^{a b}\right) \operatorname{Tr}\left(L F^{b a}\right)\right] .
$$

The last quantity is a typical reordering contribution, not to be obtained from the explicitely symmetric Weyl-ordered generators (26). One can compute its $p_{i} p_{j}(i \neq j)$ term which is $2 F_{i j} F_{j i}$ : it is non zero, dynamical and does not contain $1 /\left(q_{i}-q_{j}\right)$, hence it lies outside the original algebra $W_{H W}^{n,(s)}$.

This third remark deserves elaboration. It is nevertheless possible to define a large $N$ limit of the observable algebra in which the two sets $W_{n}^{m}$ and $W_{H W}^{n,(s)}$ are identified and then close a Poisson algebra. Redefining

$$
\begin{aligned}
W_{n}^{m} & =N^{n+m-2} \bar{W}_{n}^{m} \\
\tilde{F}_{n, m}^{a b} & =N^{n+m} \bar{F}_{n, m}^{a b}
\end{aligned}
$$

eliminates all reordering terms when $N \rightarrow \infty$, since reordering always decreases the number $n+m$ of $L$ and $Q$ terms - not forgetting that the term $\operatorname{Tr}(L \cdots Q \cdots F)$, which arises as first step in reordering of $W_{n}^{m}$ and would be of order $N^{n+m-2}$ also, is in fact reexpressed through prop.(5) as polynomials of $(L, Q)$ of length $n+m-4$, hence is actually of lower order.

In this limit:

$$
\begin{aligned}
\left\{\bar{W}_{n_{1}}^{m_{1}}, \bar{W}_{n_{2}}^{m_{2}}\right\} & =\left(n_{1} m_{2}-n_{2} m_{1}\right) \bar{W}_{n_{1}+n_{2}-1}^{m_{1}+m_{2}-1} \\
\left\{\bar{W}_{n_{1}}^{m_{1}}, \bar{F}_{n_{2}, m_{2}}^{a b}\right\} & =\left(n_{1} m_{2}-n_{2} m_{1}\right) \bar{F}_{n_{1}+n_{2}-1, m_{1}+m_{2}-1}^{a b} \\
\left\{\bar{F}_{n_{1}, m_{1}}^{a b}, \bar{F}_{n_{2}, m_{2}}^{c d}\right\} & =\delta_{a d} \bar{F}_{n_{1}+n_{2}, m_{1}+m_{2}}^{c b}-\delta_{c b} \bar{F}_{n_{1}+n_{2}, m_{1}+m_{2}}^{a d} .
\end{aligned}
$$

Indeed the first algebra realizes the structure of $\operatorname{Sdiff}\left(\mathbb{R}^{2}\right) \sim \mathcal{W}_{\infty}$, classical limit of the quantum algebra defined in [7]; the $N \rightarrow \infty$ limit achieves the decoupling of the two algebras $\mathcal{W}_{\infty}$ and $s l(r) \otimes \mathbb{C}(\lambda) \otimes \mathbb{C}(\mu)$ (double loop algebra of $\tilde{F}_{n m}^{a b}$ ).

\section{The trigonometric Euler-Calogero-Moser model}

The phase space structure is the same as before. The Hamiltonian is now:

$$
H=\frac{1}{2} \sum_{i=1}^{N} p_{i}^{2}-\frac{1}{2} \sum_{\substack{i, j=1 \\ i \neq j}}^{N} \frac{F_{i j} F_{j i}}{\sinh ^{2}\left(q_{i}-q_{j}\right)}
$$

The Lax matrix with spectral parameter takes the form:

$$
L(\lambda)=L-(\operatorname{coth}(\lambda)+1) F
$$

where

$$
\begin{aligned}
F & =\sum_{a=1}^{r} \sum_{i, j=1}^{N} \xi_{i}^{a} \eta_{j}^{a} e_{i j} \\
L & =\sum_{i=1}^{N}\left(p_{i}+F_{i i}\right) e_{i i}+2 \sum_{\substack{i, j=1 \\
i \neq j}}^{N} \frac{F_{i j}}{1-\mathrm{e}^{2\left(q_{i}-q_{j}\right)}} e_{i j}
\end{aligned}
$$


The Hamiltonian is given by $H=1 / 2\left[\operatorname{Tr}\left(L^{2}\right)-2 \alpha p-N \alpha^{2}\right]$.

As in the rational case, and for the same reason, a natural choice of observables is the set of (adinvariant) traces of monomials of the generators $\left\{L, F^{a b}, K=\exp (2 Q), K^{-1}\right\}$. They realize the following Poisson structure [5:

$$
\begin{aligned}
\{L \stackrel{\otimes}{,} L\} & =[R, L \otimes \mathbb{1}]-\left[R^{\pi}, \mathbb{1} \otimes L\right]+\sum_{\substack{i, j=1 \\
i \neq j}}^{N} \frac{F_{i i}-F_{j j}}{\sinh ^{2}\left(q_{i}-q_{j}\right)} e_{i j} \otimes e_{j i} \\
\left\{L \stackrel{\otimes}{,} K^{ \pm 1}\right\} & =-\left[R^{\pi}, \mathbb{1} \otimes K^{ \pm 1}\right] \pm\left(K^{ \pm 1} \otimes \mathbb{1}+\mathbb{1} \otimes K^{ \pm 1}\right) \mathcal{C} \\
\left\{L \stackrel{\otimes}{,} F^{a b}\right\} & =-\left[R^{\pi}, \mathbb{1} \otimes F^{a b}\right]+\left[\mathcal{C}, F^{a b}\right] \\
\left\{K^{ \pm 1} \stackrel{\otimes}{,} K^{ \pm 1}\right\} & =\left\{K^{\otimes}, K^{-1}\right\}=\left\{K^{ \pm 1} \stackrel{\otimes}{,} F^{a b}\right\}=0 \\
\left\{F^{a b} \stackrel{\otimes}{,} F^{c d}\right\} & =\left(\delta_{a d} F^{c b} \otimes \mathbb{1}-\delta_{c b} \mathbb{1} \otimes F^{a d}\right) \mathcal{C}
\end{aligned}
$$

where

$$
R=-\sum_{\substack{i, j=1 \\ i \neq j}}^{N} \operatorname{coth}\left(q_{i}-q_{j}\right) e_{i j} \otimes e_{j i},
$$

$\mathcal{C}$ still being the Casimir element of $\operatorname{sl}(N)$.

The commutation relations involved in the normal-ordering procedure become (with $F_{i i}=\alpha$ ):

$$
\begin{aligned}
{[L, K] } & =2 F K-2 \alpha K \\
{\left[L, K^{-1}\right] } & =-2 K^{-1} F+2 \alpha K^{-1} .
\end{aligned}
$$

The algebra of relevant, algebraically independant observables, follows from prop.(3) and the two equivalents of prop.(勾) and (5) describing normal-ordering. Here however both positive and negative powers of $K$ are allowed.

Proposition 6 All monomials $\operatorname{Tr}\left(A^{(1)} \cdots A^{(n)}\right)$ and $\operatorname{Tr}\left(A^{(1)} \cdots A^{(n)} F^{a b}\right)$ with $A \in\left\{L, K, K^{-1}\right\}$ can be rewritten as polynomials of normal-ordered generators $\operatorname{Tr}\left(L^{p} K^{q}\right)$ and $\operatorname{Tr}\left(L^{p} K^{q} F^{c d}\right) \equiv F_{p, q}^{c d}, p \in \mathbb{N}, q \in \mathbb{Z}$.

Proof. similar as prop.(4). From relations (41) and (42), commuting $L$ through $K$ or $K^{-1}$ to normalorder decreases by 1 the number of $L$ terms and creates an $F$ term. Hence a recursion proof on the total number of $L$ terms is derived straightforwardly.

Proposition 7 Monomials of the form $\operatorname{Tr}\left(L^{n} K^{m} F\right)$ with $m \neq 0$ can be rewritten as polynomials of the variables $\operatorname{Tr}\left(L^{p} K^{q}\right)$ and $\operatorname{Tr}\left(L^{p} K^{q} \tilde{F}^{a b}\right)$.

Proof. As in prop.(5), the commutation relations (41, 42) allow to define a recursive procedure.

- When the length (i.e., the number of $L$ terms) is 0 ,

$$
\begin{aligned}
\operatorname{Tr}(K F) & =\frac{1}{2} \operatorname{Tr}([L, K]+2 \alpha K)=\alpha \operatorname{Tr}(K) \\
\operatorname{Tr}\left(K^{-1} F\right) & =-\frac{1}{2} \operatorname{Tr}\left(\left[L, K^{-1}\right]-2 \alpha K^{-1}\right)=\alpha \operatorname{Tr}\left(K^{-1}\right) .
\end{aligned}
$$

- We assume the proposition to be true up to the length $n-1$. One has for $m \neq 0$ :

$$
0=\operatorname{Tr}\left[L, L^{n} K^{m}\right]=2 m \operatorname{Tr}\left(L^{n} K m F\right)-2 \alpha m \operatorname{Tr}\left(L^{n} K^{m}\right)+\text { ordering terms, }
$$

with ordering terms of length $n-1$ which are then written using prop.( (5) and recursion hypothesis. This proof collapses when $m=0$. 
Hence our generators are chosen to be

$$
\begin{aligned}
W_{n}^{m} & =\operatorname{Tr}\left(L^{n} K^{m}\right) \\
F_{n}^{a b} & =\operatorname{Tr}\left(L^{n} F^{a b}\right)=F_{n, o}^{a b} \\
\tilde{F}_{n, m}^{a b} & =\operatorname{Tr}\left(L^{n} K^{m} \tilde{F}^{a b}\right)
\end{aligned}
$$

The Poisson algebra of observables now follows from (36) and (43):

$$
\begin{aligned}
& \left\{W_{n_{1}}^{m_{1}}, W_{n_{2}}^{m_{2}}\right\}=\quad \sum_{i=1}^{n_{1}} \sum_{j=1}^{m_{2}} \operatorname{Tr}\left(L^{n_{1}-i} K^{m_{1}} L^{i-1}\left(K^{m_{2}-j+1} L^{n_{2}} K^{j-1}+K^{m_{2}-j} L^{n_{2}} K^{j}\right)\right) \\
& -\sum_{i=1}^{m_{1}} \sum_{j=1}^{n_{2}} \operatorname{Tr}\left(\left(K^{m_{1}-i} L^{n_{1}} K^{i}+K^{m_{1}-i+1} L^{n_{1}} K^{i-1}\right) L^{n_{2}-j} K^{m_{2}} L^{j-1}\right) \\
& =2\left(n_{1} m_{2}-n_{2} m_{1}\right) W_{n_{1}+n_{2}-1}^{m_{1}+m_{2}}+\text { ordering terms } \\
& \left\{W_{n_{1}}^{m_{1}}, F_{n_{2}, m_{2}}^{a b}\right\}=\quad \sum_{i=1}^{n_{1}} \sum_{j=1}^{m_{2}} \operatorname{Tr}\left(L^{n_{1}-i} K^{m_{1}} L^{i-1}\left(K^{m_{2}-j+1} F^{a b} L^{n_{2}} K^{j-1}+K^{m_{2}-j} F^{a b} L^{n_{2}} K^{j}\right)\right) \\
& -\sum_{i=1}^{m_{1}} \sum_{j=1}^{n_{2}} \operatorname{Tr}\left(\left(K^{m_{1}-i} L^{n_{1}} K^{i}+K^{m_{1}-i+1} L^{n_{1}} K^{i-1}\right) L^{n_{2}-j} K^{m_{2}} F^{a b} L^{j-1}\right) \\
& +\sum_{i=1}^{n_{1}} \operatorname{Tr}\left(\left[L^{n_{2}} K^{m_{2}}, L^{n_{1}-i} K^{m_{1}} L^{i-1}\right] F^{a b}\right) \\
& =2\left(n_{1} m_{2}-n_{2} m_{1}\right) F_{n_{1}+n_{2}-1, m_{1}+m_{2}}^{a b}+\text { ordering terms } \\
& \left\{F_{n_{1}, m_{1}}^{a b}, F_{n_{2}, m_{2}}^{c d}\right\}=\quad \sum_{i=1}^{n_{1}} \sum_{j=1}^{m_{2}} \operatorname{Tr}\left(L^{n_{1}-i} K^{m_{1}} F^{a b} L^{i-1}\left(K^{m_{2}-j+1} F^{c d} L^{n_{2}} K^{j-1}+K^{m_{2}-j} F^{c d} L^{n_{2}} K^{j}\right)\right) \\
& -\sum_{i=1}^{m_{1}} \sum_{j=1}^{n_{2}} \operatorname{Tr}\left(\left(K^{m_{1}-i} F^{a b} L^{n_{1}} K^{i}+K^{m_{1}-i+1} F^{a b} L^{n_{1}} K^{i-1}\right) L^{n_{2}-j} K^{m_{2}} F^{c d} L^{j-1}\right) \\
& +\sum_{i=1}^{n_{1}} \operatorname{Tr}\left(L^{n_{1}-i} K^{m_{1}} F^{a b} L^{i-1}\left[F^{c d}, L^{n_{2}} K^{m_{2}}\right]\right) \\
& -\sum_{i=1}^{n_{2}} \operatorname{Tr}\left(\left[F^{a b}, L^{n_{1}} K^{m_{1}}\right] L^{n_{2}-i} K^{m_{2}} F^{c d} L^{i-1}\right) \\
& +\delta_{a d} \operatorname{Tr}\left(L^{n_{2}} K^{m_{2}} L^{n_{1}} K^{m_{1}} F^{c b}\right)-\delta_{c b} \operatorname{Tr}\left(L^{n_{1}} K^{m_{1}} L^{n_{2}} K^{m_{2}} F^{a d}\right) .
\end{aligned}
$$

For the sake of simplicity we have expressed the last Poisson bracket (46) in terms of unreduced generators $F_{n, m}^{a b}$ instead of $\tilde{F}_{n, m}^{a b}$ for $m \neq 0$. When expressed in terms of the exact generators $\tilde{F}_{n, m}^{a b}$, expressions of analogous form to (14,15) arise. (45) is not modified and (46) acquires extra terms of the form (15). Again, some Poisson brackets do not generate reordering terms; this is the case whenever only one term $(L$ or $K)$ is present in the monomial. In particular:

$$
\begin{aligned}
& \left\{W_{n}^{0}, W_{m}^{0}\right\}=0 \quad: \text { commuting Hamiltonians } \\
& \left\{W_{n}^{0}, W_{m}^{1}\right\}=2 n W_{n+m-1}^{1} \\
& \left\{W_{n}^{0}, W_{0}^{m}\right\}=2 n m W_{n-1}^{m}
\end{aligned}
$$

but $\left\{W_{n}^{1} \stackrel{\otimes}{,} W_{m}^{1}\right\}=W_{n+m-2}^{2}+$ ordering terms. 
As previously derived, the Yangian algebra is also exact [5]:

$$
\begin{aligned}
\left\{W_{n}^{0}, F_{m, 0}^{a b}\right\}= & 0 \\
\left\{F_{n, 0}^{a b}, F_{m, 0}^{c d}\right\}= & \delta_{a d} F_{n+m, 0}^{c b}-\delta_{c b} F_{n+m, 0}^{a d} \\
& +\sum_{i=1}^{n}\left(F_{n+m-i, 0}^{c b} F_{i-1,0}^{a d}-F_{n-i, 0}^{c b} F_{m+i-1,0}^{a d}\right)+\sum_{i=1}^{m}\left(F_{n+i-1,0}^{c b} F_{m-i, 0}^{a d}-F_{i-1,0}^{c b} F_{m+n-i, 0}^{a d}\right) .
\end{aligned}
$$

It is also important to note, as in the case of rational potentials, the existence of $N$ commuting Hamiltonians with an external field, defined as:

$$
H^{(n)}=\frac{1}{2 n} \operatorname{Tr}\left(L^{+} L^{-}\right)^{n} \quad \text { with } L^{ \pm}=L \pm a K \pm b K^{-1}
$$

since the Poisson structure for $\left(L^{+} L^{-}\right)$has the same characteristic form as in the rational case, which was generically defined in [3]:

$$
\begin{aligned}
\left\{L^{+} L^{-} \stackrel{\otimes}{,} L^{+} L^{-}\right\}= & {\left[\mathcal{R}, L^{+} L^{-} \otimes \mathbb{1}\right]-\left[\mathcal{R}^{\pi}, \mathbb{1} \otimes L^{+} L^{-}\right] } \\
- & 2 a \mathcal{C}\left(L^{+} L^{-} \otimes K-K \otimes L^{+} L^{-}+L^{+} K L^{-} \otimes \mathbb{1}-\mathbb{1} \otimes L^{+} K L^{-}\right) \\
+ & 2 b \mathcal{C}\left(L^{+} L^{-} \otimes K^{-1}-K^{-1} \otimes L^{+} L^{-}+L^{+} K^{-1} L^{-} \otimes \mathbb{1}-\mathbb{1} \otimes L^{+} K^{-1} L^{-}\right)
\end{aligned}
$$

with

$$
\mathcal{R}=\left(\mathbb{1} \otimes L^{+}\right) R+R\left(\mathbb{1} \otimes L^{-}\right) .
$$

This relation is known to ensure commutation of the traces $\operatorname{Tr}\left(L^{+} L^{-}\right)^{n}$ [3]. Such traces are naturally (polynomial) functions of the observables $\operatorname{Tr}\left(L^{n} K^{m}\right)$ and $\operatorname{Tr}\left(L^{n} K^{m} F^{a b}\right)$, but it is much easier to see their commutitativity under the $L^{ \pm}$form. The field-extended models are therefore "partially" integrable. They were considered also in 11].

Again one can define a $N \rightarrow \infty$ limit for this algebra, normalizing each generator by the power of $L$ (the power of $K$ is conserved hence it is irrelevant, which is obvious since $K=\exp (2 Q)$ is anyway dimensionless):

$$
\begin{aligned}
W_{n}^{m} & =N^{n-1} \bar{W}_{n}^{m} \\
\tilde{F}_{n, m}^{a b} & =N^{n} \bar{F}_{n, m}^{a b} .
\end{aligned}
$$

All reordering terms are eliminated; unfortunately the subleading terms which were contributing to the Yangian structure are also eliminated and the remaining structure is a simple $\mathcal{W}_{\infty}$-plus-current algebra. Interestingly, the $\mathcal{W}_{\infty}$ part is the algebra $\operatorname{Sdiff}\left(\mathbb{R} \times \mathrm{S}^{1}\right)$ :

$$
\left\{\bar{W}_{n_{1}}^{m_{1}}, \bar{W}_{n_{2}}^{m_{2}}\right\}=2\left(n_{1} m_{2}-n_{2} m_{1}\right) \bar{W}_{n_{1}+n_{2}-1}^{m_{1}+m_{2}} .
$$

However, this algebra can be redefined as $\operatorname{Siff} f\left(\mathbb{R}^{2}\right)$ by the change of indexation

$$
\mathcal{W}_{n}^{n+m}=\bar{W}_{n}^{m} \text {. }
$$

In particular the trigonometric Hamiltonian $\bar{W}_{2}^{0}=1 / N \operatorname{Tr}\left(L^{2}\right)$ is identified with the rational generator $\mathcal{W}_{2}^{2} \sim \operatorname{Tr}\left(L^{2} Q^{2}\right)$. This identification was mentioned in the large $N$ limit in [7]. Similarly all commuting trigonometric Hamiltonians become (for $N \rightarrow \infty$ ) particular rational observables - an interesting algebraic connection, though yet restricted to $N \rightarrow \infty$.

\section{Conclusion}

We have explicitly constructed polynomial algebras of observables for the ECM models, both rational and trigonometric. Due to their high degree of intricacy, they do not seem to help in directly solving the model - as could be hoped from [7]- when $N$ is finite. They provide us however with a totally unified structure for the commuting Hamiltonians, the current-algebra or Yangian-symmetry generators, and an interesting 
Virasoro algebra of "intertwiners" in the rational case. They also suggest an algebraic relation between the rational and trigonometric cases though yet only achieved for infinite $N$.

The case of ECM models in external fields is more hopeful. Indeed one expects as in the case of pure CM models [3, 12 to extract from the algebra of observables - expressed in the suitable $\left\{L^{+}, L^{-}\right\}$basisexact eigenfunctions of the Hamiltonian flows, at least the $N$ commuting flows yet known.

Acknowledgements We wish to thank O. Babelon and D. Bernard for discussions. J.A. thanks P. Sorba for his hospitality at LPT, ENS Lyon. Part of this work was done at SISSA Trieste under EEC contract 540081.

\section{References}

[1] F. Calogero, Lett. Nuovo Cimento 13 (1975) 411; Lett. Nuovo Cimento 16 (1976) 77; J. Math. Phys. 12 (1971) 419; J. Moser, Adv. Math. 16 (1976) 1; B. Sutherland, Phys. Rev. Lett. 34 (1975) 1083.

[2] J. Gibbons and T. Hermsen, Physica 11D (1984) 337; S. Wojciechowski, Phys. Lett. $111 \mathrm{~A}$ (1985) 101.

[3] J. Avan, Phys. Lett. 185 A (1994) 293.

[4] K. Hikami and M. Wadati, J. Phys. Soc. Jpn. 62 (1993) 4203; hep-th 9402026; H. Ujino, K. Hikami and M. Wadati, J. Phys. Soc. Jpn. 61 (1992) 3425; 62 (1993) 3035.

[5] E. Billey, J. Avan and O. Babelon, hep-th/9401117 (to appear in Phys. Lett. A).

[6] D. Bernard, M. Gaudin, F.D.M. Haldane and V. Pasquier, J. Phys. A: Math. Gen. 26 (1993) 5219.

[7] K. Hikami and M. Wadati, preprint (1994).

[8] E.K. Sklyanin, "On the complete integrability of the Landau-Lifchitz equation." preprint LOMI E-3-79 Leningrad 1979.

[9] M.A. Olshanetskii and A.M. Perelomov, Inv. Math. 37 (1976) 76.

[10] E. Billey, J. Avan and O. Babelon, Phys. Lett. A 186 (1994) 114.

[11] A.P. Polychronakos, Phys. Rev. Lett. 69 (1992) 703; J. Minahan and A.P. Polychronakos, Phys. Lett. 302 B (1993) 265.

[12] A.M. Perelomov, Sov. Journ. Part. Nucl. 10 (1979) 336. 